\title{
Evaluer la diversité du paysage des pâturages africains
}

\author{
M. Godron ${ }^{1}$ J. Poissonet ${ }^{2}$ Ph. Daget ${ }^{2}$
}

Mots-clés

Pâturages - Mesure - Méthode Paysage - Zone tropicale.

\section{Résumé}

La diversité d'un paysage peut être mesurée par une application de la théorie de l'information à l'analyse d'un panorama découpé en plans selon les facettes géomorphologiques et les types de végétation. Des applications sont proposées sur des paysages d'Afrique tropicale septentrionale.

\section{INTRO DUCTION}

Il est souvent écrit que l'ensemble des paysages d'Afrique est très divers. Il est plus difficile de dire dans quelle mesure chacun de ces paysages est divers ou, au contraire, uniforme. C'est tout le problème de la qualification de la diversité et de la valeur des paysages d'Afrique qui est ainsi posé, mais l'expérience montre que les critères qui permettent de juger de la qualité d'un paysage naturel sont rarement précisés. Cette diversité dépend de nombreux paramètres tels que, par exemple, le nombre et la nature des principaux éléments qui peuvent être retenus : arbres, herbes, fleurs, animaux, maisons, rochers, eau, relief, leur forme ou leur couleur.

En face d'un paysage de savane ou de steppe, l'importance accordée à ces paramètres sera variable suivant les individus et le niveau de perception. Heureusement, il existe une méthode $(1,3)$ offrant la possibilité d'en analyser la diversité en fonction de paramètres simples. On examine le nombre et la disposition des «plans» déterminés par le relief du sol et par la végétation, au sens où l'on parle en photographie du premier plan, du second plan.

C'est cette méthode qui va être reprise ici sur des exemples plus familiers aux africanistes que ceux qui illustraient le travail original.

\section{PRIN CIPES ET METHODES}

Pour être plus précise, l'analyse sera limitée à une fraction du paysage correspondant à celle qui entre dans le champ d'un appareil photographique de focale normale (soit $50 \mathrm{~mm}$ pour un appareil de format 24 x 36). La présence ou l'absence de chaque plan (au sens

1. Institut de Botanique de l'Université Montpellier II, Montpellier, France actuellement La Graineterie, 18410 Brinon sur Sauldre, France ;

E-mail : michel.godron@ libertysurf.net

2. Cirad-emvt/Cnrs, TA 30/F, Campus international de Baillarguet, 34398 Montpellier Cedex 5, France ; E-mail : philippe.daget@cirad.fr de « premier plan », « deuxième plan », déterminés par les facettes du relief et de la végétation) sera examinée dans chaque maille d'une grille de « carrés » contigus tracés sur la photographie. Ce qui revient à répondre à la question : le plan A est-il présent dans la maille ? Chacune des réponses apporte, selon la définition classique de la théorie de l'information (2), une unité d'information appelée shannon (autrefois binon) ; quand on examine plusieurs carrés, l'information recueillie croît comme le logarithme du nombre de cas possibles, équiprobables à priori.

Considérons d'abord un plan A et 8 carrés contigus en deux rangées. Si le plan A n'est présent qu'une seule fois, il peut occuper 8 positions différentes (figure 1) et l'information correspondante est, par définition, égale à :

$$
\mathrm{I}=\log _{2} 8=3 \text { shannons }
$$

Puis le plan $B$ présent dans deux mailles peut l'être de 28 manières différentes (figure 2) et l'information correspondante est égale à :

$$
\mathrm{I}=\log _{2} 28=4,8 \text { shannons }
$$

Si les 8 positions du plan A et les 28 positions du plan B sont combinées entre elles, on obtient $8 \times 28=224$ cas possibles. L'information correspondante devient :

$$
\mathrm{I}=\log _{2} 224=7,8 \text { shannons }
$$

On vérifie bien que l'information relative au plan A s'ajoute à celle du plan B, puisque :

$$
\log _{2} 224=\log _{2}(8 \times 28)=\log _{2} 8+\log _{2} 28=3+4,8=7,8
$$

Plus généralement, si le plan A est présent $\mathrm{A}$ fois dans $\mathrm{S}$ mailles, le nombre de cas possibles est :

$$
\mathrm{C}_{\mathrm{S}}^{\mathrm{A}}=\frac{\mathrm{S} !}{\mathrm{A} !(\mathrm{S}-\mathrm{A}) !}
$$

et l'information est, avec les mêmes conventions :

$$
\mathrm{I}_{\mathrm{A}}=\log _{2} \mathrm{C}_{\mathrm{S}}^{\mathrm{A}}
$$



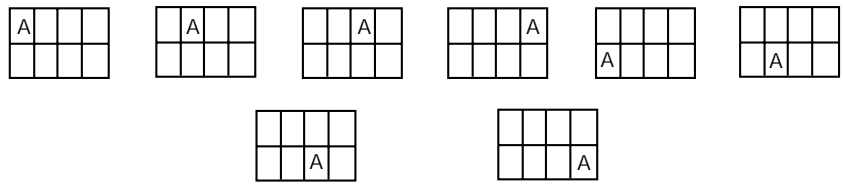

Figure 1 : le plan $A$, présent une seule fois, peut occuper 8 positions.

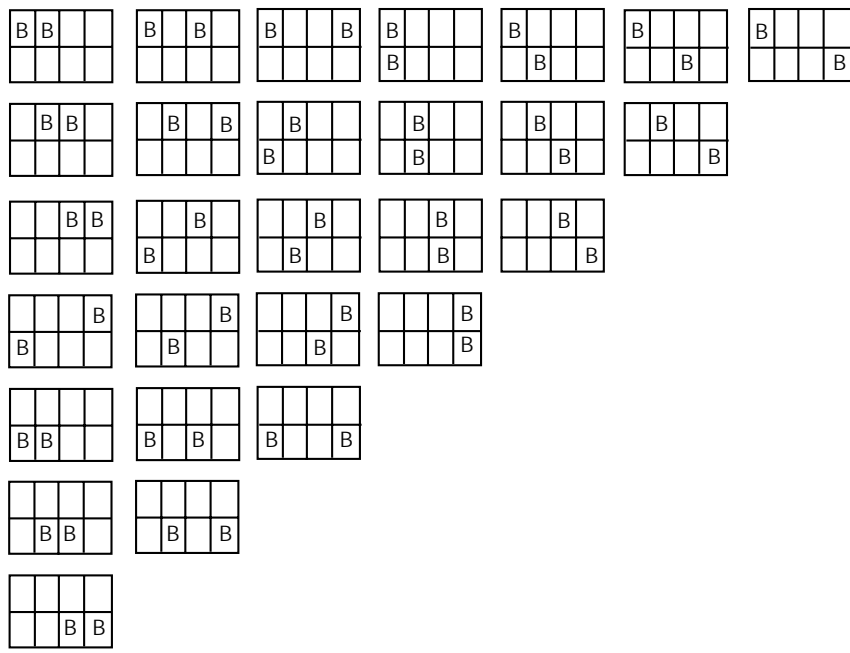

Figure 2 : le plan B est présent dans 2 carrés, d'où 28 combinaisons possibles.

En définitive, pour l'ensemble des plans A, B, C ... J, présents sur une photographie, l'information totale est :

$$
\mathrm{I}=\mathrm{I}_{\mathrm{A}}+\mathrm{I}_{\mathrm{B}}+\mathrm{I}_{\mathrm{C}}+\ldots+\mathrm{I}_{\mathrm{J}}
$$

Si le plan A n'est pas présent sur le cliché, ou s'il est présent dans toutes les mailles, il n'apporte aucune diversité au paysage :

$$
\mathrm{C}_{8}^{0}=1 \text { et } \mathrm{I}_{\mathrm{A}}=\log 1=0
$$

Si le plan A est présent dans une seule des 8 mailles, il n'apporte que peu de diversité et l'on peut écrire :

$$
\mathrm{C}_{8}^{1}=8 \text { et } \mathrm{I}_{\mathrm{A}}=\log 8=3
$$

Si le plan A est présent dans 2 mailles, il apporte un peu plus de diversité :

$$
\mathrm{C}_{8}^{2}=\frac{8 !}{2 !(8-2) !}=\frac{8 \times 7}{2}=28 \text { et } \log _{2} 28=4,8
$$

Au fur et à mesure que le nombre de présences du plan A dans les mailles augmente, $\mathrm{I}_{\mathrm{A}}$ augmente aussi, tant que le nombre de présences est inférieur à $\mathrm{S} / 2$, ici à 4 ; ensuite il diminue. Enfin, si le plan A est présent partout, la photo est uniforme en ce qui le concerne et donc non diversifiée, alors :

$$
\mathrm{C}_{8}^{8}=1 \text { et } \mathrm{I}_{\mathrm{A}} \log _{2} 1=0
$$

En définitive :

un plan présent dans 0 ou 8 mailles donne 0 shannon un plan présent dans 1 ou 7 mailles donne 3 shannons un plan présent dans 2 ou 6 mailles donne 4,8 shannons

un plan présent dans 3 ou 5 mailles donne 5,8 shannons

un plan présent dans 4 mailles donne 6,1 shannons

Soit I la somme des résultats correspondant aux différents plans. Plus la photo - donc le paysage - est diversifiée, plus I est grand ; en revanche, plus la photo et le paysage sont uniformes, plus I est petit. C'est donc cette quantité I qui mesure la diversité globale de la fraction du paysage retenue sur le cliché.

\section{APPLICATIONS}

\section{Calcul de la diversité globale de paysages d'Afrique}

Pour calculer la diversité globale d'un paysage, il suffit :

a) de délimiter et de dénombrer les différents plans sur la photographie ;

b) de placer sur le cliché une grille de rectangles contigus en deux rangs ; par commodité, on en met 8 sur un cliché en focale normale (mais il est préférable d'en prendre 12 sur un cliché panoramique);

c) de compter le nombre de mailles où chacun des plans est présent ;

d) de se reporter au tableau précédent pour avoir le nombre de shannons (dans le cas de 8 mailles; pour 12 il faut établir un tableau équivalent);

e) de faire la somme des valeurs obtenues.

Quelques exemples détaillés montreront la manière de procéder.

\section{Une savane ouverte du Tchad}

La première photo, prise dans une savane ouverte de l'est du Tchad, est interprétée sur un schéma (figure 3). Trois plan apparaissent. Le plan 1 est présent dans 8 mailles sur 8 , le 2 et le 3 dans 4 mailles sur 8 . Par conséquent :

$$
\mathrm{I}_{1}=0 ; \mathrm{I}_{2}=6,1 ; \mathrm{I}_{3}=6,1
$$

Pour le paysage :

$$
\mathrm{I}=0+6,1+6,1=12,2
$$

La diversité de ce paysage tchadien est donc de 12,2 shannons.

\section{Une savane du Cameroun}

La deuxième photo correspond à une savane humide altitudinale de l'Adamaoua au Cameroun. Son interprétation sur la figure 4 montre que les plans 1 et 2 sont présents dans 4 mailles, les plans 3 et 4 dans 6 mailles et le plan 5 dans 5 mailles. Par conséquent, la diversité globale de ce paysage de montagne est :

$$
I=(2 \times 6,1)+(2 \times 4,8)+5,8=27,6
$$

\section{Un paysage du Hoggar}

La troisième photo montre un aspect du panorama visible du seuil de l'oratoire du Père de Foucauld au sommet du Hoggar en Algérie. La figure 5 est son interprétation sous l'angle des plans. Le tableau I en donne la ventilation.

Soit au total pour le paysage :

$$
\mathrm{I}=\sum_{11} \mathrm{I}_{\mathrm{n}}=55,5
$$




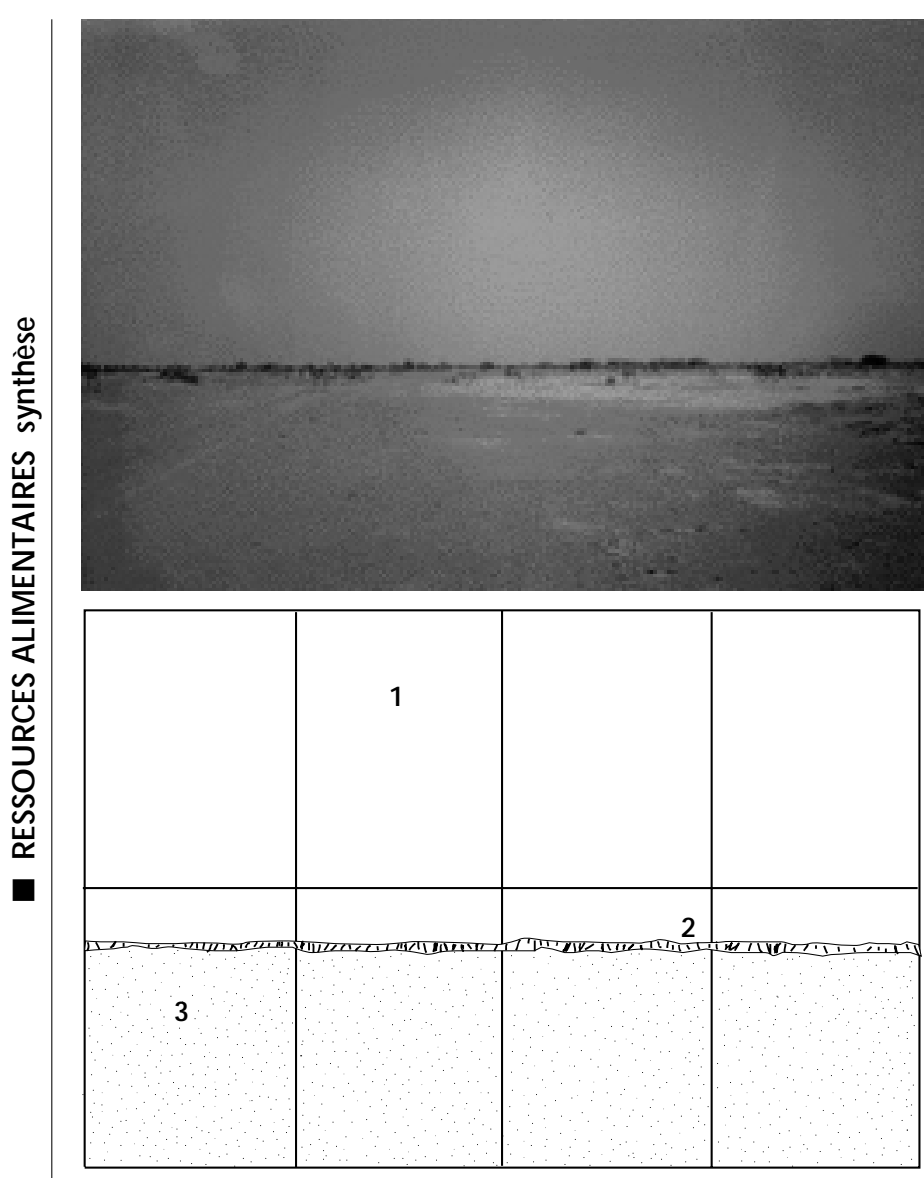

Figure 3 : photo d'une savane ouverte de l'est du Tchad (. Poissonet) et interprétation du paysage.
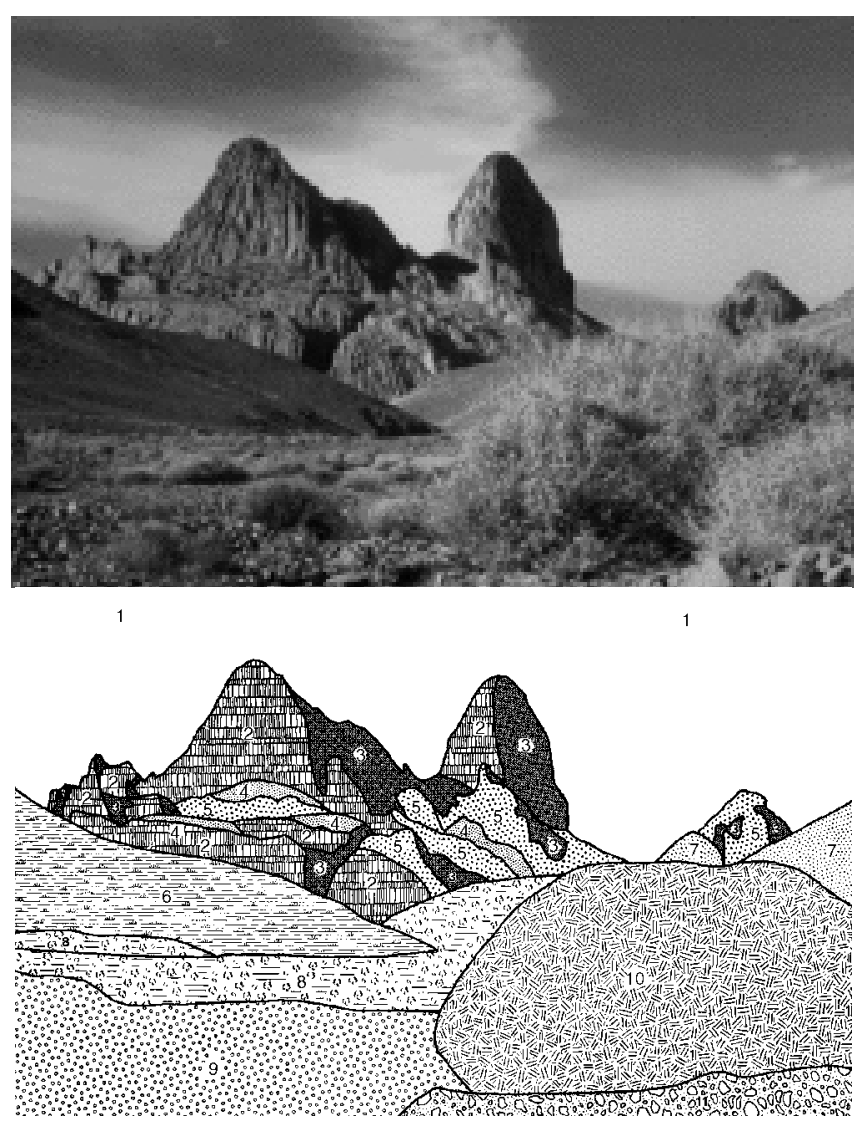

Figure 5 : photo de savanes altitudinales du Hoggar en Algérie (N. Daget) et interprétation du paysage.
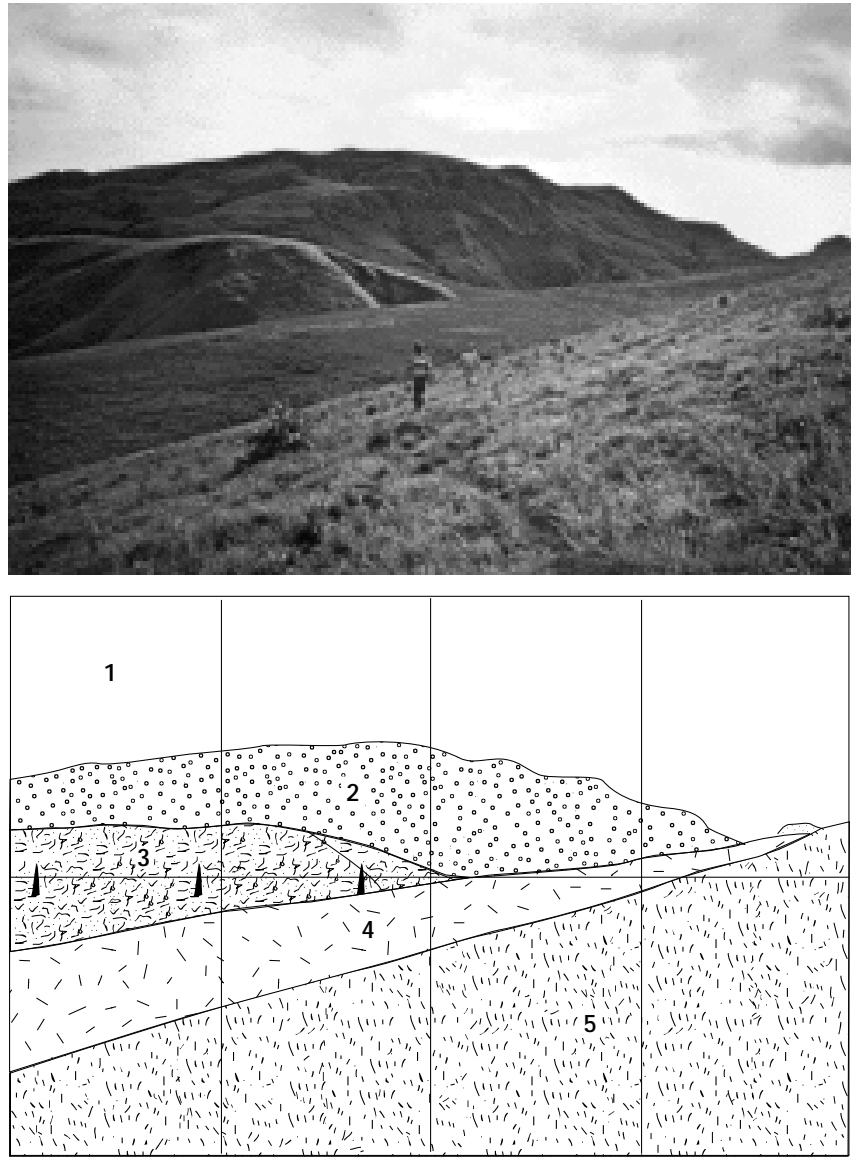

Figure 4 : photo d'une savane humide de I'Adamaoua au Cameroun (Cirad-forêt) et interprétation du paysage.

\section{Critère de jugement}

La diversité globale calculée I permet de porter un jugement objectif sur la diversité d'ensemble du paysage visionné. L'échelle suivante en donne le moyen :

$$
\begin{aligned}
& \text { de } 0 \text { à } 10 \text { : paysage très faiblement diversifié } \\
& \text { de } 11 \text { à } 20 \text { : paysage faiblement diversifié } \\
& \text { de } 21 \text { à } 30 \text { : paysage moyennement diversifié } \\
& \text { de } 31 \text { à } 40 \text { : paysage fortement diversifié } \\
& \text { de } 41 \text { à } 50 \text { : paysage très fortement diversifié } \\
& \text { plus de } 51 \text { : paysage extrêmement diversifié }
\end{aligned}
$$

Pour les trois cas présentés ici, le paysage du Tchad est très faiblement diversifié, celui du Cameroun est moyennement diversifié et celui du Hoggar extrêmement diversifié.

\section{Diversité relative}

\section{Moyenne des plans du paysage}

La diversité moyenne des plans du paysage est définie comme le quotient de la diversité globale telle qu'elle vient d'être calculée par le nombre de plans retenus dans l'interprétation de la photographie témoin du paysage. Ainsi, pour les trois exemples cités, on a respectivement :

$\begin{array}{lrrl}\text { Tchad } & 12,2 & 3 & \mathrm{Dm}=12,2 / 3=4,1 \\ \text { Cameroun } & 27,6 & 5 & \mathrm{Dm}=27,6 / 5=5,5 \\ \text { Algérie } & 55,5 & 11 & \mathrm{Dm}=55,5 / 11=5\end{array}$


Tableau I

Eléments de l'interprétation de la diversité du panorama du Hoggar

\begin{tabular}{|c|c|c|c|c|c|c|c|c|c|c|}
\hline \multirow{2}{*}{$\begin{array}{l}\text { Plans } \\
\text { n }\end{array}$} & \multicolumn{8}{|c|}{ Carrés } & \multirow[t]{2}{*}{$\Sigma$} & \multirow[t]{2}{*}{$I_{n}$} \\
\hline & 1 & 2 & 3 & 4 & 5 & 6 & 7 & 8 & & \\
\hline 1 & $x$ & $x$ & $x$ & $x$ & & & $x$ & $x$ & 6 & 4,8 \\
\hline 2 & $x$ & $x$ & $x$ & & $x$ & $x$ & & & 5 & 5,8 \\
\hline 3 & $x$ & $x$ & $x$ & $x$ & & $x$ & $x$ & $x$ & 7 & 3,0 \\
\hline 4 & $x$ & $x$ & $x$ & & & $x$ & $x$ & & 5 & 5,8 \\
\hline 5 & $x$ & $x$ & $x$ & $x$ & & $x$ & $x$ & $x$ & 7 & 3,0 \\
\hline 6 & $x$ & $x$ & & & $x$ & $x$ & & & 4 & 6,1 \\
\hline 7 & & & & $x$ & & & & $x$ & 2 & 4,8 \\
\hline 8 & & & & & $x$ & $x$ & $x$ & & 3 & 5,8 \\
\hline 9 & & & & & $x$ & $x$ & $x$ & & 3 & 5,8 \\
\hline 10 & & & & & & & $x$ & $x$ & 2 & 4,8 \\
\hline 11 & & & & & & $x$ & $x$ & $x$ & 3 & 5,8 \\
\hline
\end{tabular}

\section{Diversité maximale d'un paysage}

C'est la diversité qui serait atteinte si chaque plan de la photographie témoin était présent dans une maille sur deux. Elle est égale à :

$$
\mathrm{I}_{\max }=\mathrm{N} \log _{2} \mathrm{C}_{\mathrm{S}}^{\mathrm{S} / 2}
$$

où $\mathbf{S}$ est le nombre de mailles et $\mathbf{N}$ le nombre de plans; dans le cas particulier présent où $\mathrm{S}=8$, la diversité maximale par plan est égale à :

$$
\mathrm{C}_{8}^{4}=6,1
$$

et celle du paysage à :

$$
\mathrm{I}_{\max }=6,1 \mathrm{~N}
$$

Par conséquent, pour les trois paysages africains examinés ici, la diversité maximale est respectivement de :

\section{8,3 pour le paysage du Tchad \\ 30,5 pour celui du Cameroun \\ 67,1 pour celui de l'Algérie}

\section{Diversité relative d'un paysage}

Connaissant la diversité globale effective et la diversité maximale, il est possible de caractériser la diversité relative des paysages par le rapport :

$$
\mathrm{Q}=\frac{\mathrm{I}}{\mathrm{I}_{\max }}
$$

Le barème suivant permet un jugement objectif de la diversité réalisée :

$$
\begin{aligned}
& \mathrm{Q}<0,5 \\
& 0,5<\mathrm{Q}<0,6 \\
& 0,6<\mathrm{Q}<0,7 \\
& 0,7<\mathrm{Q}<0,8 \\
& 0,8<\mathrm{Q}<0,9 \\
& \mathrm{Q}>0,9
\end{aligned}
$$

paysage de diversité relative très médiocre paysage de diversité relative médiocre paysage de diversité relative moyenne paysage de diversité relative bonne paysage de diversité relative très bonne paysage de diversité relative excellente
Pour les trois paysages examinés ici, on a :

$$
\mathrm{Q}(1)=0,67 ; \mathrm{Q}(2)=0,90 ; \mathrm{Q}(3)=0,83
$$

Donc le paysage tchadien présente une diversité relative moyenne, en ce sens qu'avec seulement 3 plans la diversité atteinte est relativement réduite. En revanche, celle du Cameroun est excellente, ce qui veut dire qu'avec 9 plans la diversité atteinte est très proche du

\begin{tabular}{|c|c|c|c|c|}
\hline \multirow[t]{2}{*}{ Paysage } & \multirow[t]{2}{*}{ Nb. de plans } & \multicolumn{3}{|c|}{ Diversité } \\
\hline & & Effective & Maximale & Relative \\
\hline $\mathrm{H} 2$ & 11 & 49 & 67,1 & 0,73 \\
\hline M 4 & 9 & 37,6 & 54,9 & 0,68 \\
\hline K & 9 & 51,7 & 54,9 & 0,94 \\
\hline G & 4 & 16,7 & 24,4 & 0,68 \\
\hline D & 4 & 24,1 & 16,7 & 0,98 \\
\hline$S$ & 5 & 28,9 & 30,5 & 0,94 \\
\hline $\mathrm{R}$ & 5 & 21 & 30,5 & 0,68 \\
\hline$A$ & 8 & 34,6 & 48,8 & 0,70 \\
\hline$P$ & 7 & 34,7 & 42,7 & 0,81 \\
\hline$F$ & 5 & 26,1 & 30,5 & 0,85 \\
\hline C & 6 & 27,6 & 36,6 & 0,85 \\
\hline V & 6 & 29,1 & 36,6 & 0,79 \\
\hline W & 7 & 41,8 & 42,7 & 0,97 \\
\hline B & 7 & 34,9 & 42,7 & 0,81 \\
\hline 0 & 7 & 42,1 & 42,7 & 0,98 \\
\hline U & 6 & 28,9 & 36,6 & 0,78 \\
\hline
\end{tabular}
maximum possible. Celle du Hoggar est très bonne, donc assez proche du maximum possible avec 11 plans.

\section{Un cas concret : les paysages de l'Est tchadien}

Poissonet et coll. (5) ont reconnu 57 « unités pastorales » dans la partie du territoire tchadien situé entre 9 et $16^{\circ} \mathrm{N}$ et entre 19 et $23^{\circ} \mathrm{E}$; elles sont regroupées en 18 « unités de végétation », ellesmêmes réunies en 8 «grandes unités géomorphologiques ». Seize

Tableau II

Eléments de l'appréciation de la diversité des principaux paysages de I'Est tchadien 
des 18 unités de végétation sont illustrées par la photographie d'un panorama-type. Ces 16 clichés ont été réinterprétés sous l'aspect de leur diversité (tableau II).

Dans un graphique portant la diversité effective en fonction de la diversité maximale (figure 6), les images des 16 paysages se répartissent en cinq groupes de diversité effective décroissante, depuis les milieux sub-désertiques jusqu'aux milieux montagneux :

I : trois unités de végétation des ensablements dunaires et regs sahéliens, D, G et R ;

II : un groupe intermédiaire avec les unités des végétations herbacées des dépôts sableux $(\mathrm{S})$ et steppes boisées sur cuirasse $(\mathrm{F})$;

III : les unités des plaines et vallées, A, B, C, M, P, V avec l'unité culture $\mathrm{U}$; on peut distinguer deux sous-groupes : en bas, avec les cultures, les steppes plus ou moins boisées à épineux sous climat sahélien et en haut les steppes boisées et forêts claires du Salamat sous climat soudanien ;

IV : la végétation hydrophile soudanienne, mares $(\mathrm{O})$ et plaines $(\mathrm{W})$;

$\mathrm{V}$ : deux unités des massifs et reliefs, $\mathrm{H}$ et $\mathrm{K}$.

Chacun de ces groupes correspond à une « famille » de paysage au sens de Papillon et Godron (4). On note une bonne corrélation entre les deux types de diversité $(r=0.92 * * *)$ indiquant qu'en première approximation la diversité effective d'un paysage est voisine de $73 \%$ de sa diversité maximale.

\section{CONCLUSION}

Le procédé d'analyse qui vient d'être rappelé donne un moyen simple et rapide de caractériser objectivement la diversité d'un paysage. Il peut conduire à des approches plus raffinées prenant en compte la couleur des plans ou leur dimensions et leur agencement. Comme la diversité d'une végétation est variable d'un emplacement à un autre, un angle de vue même légèrement différent peut produire une valeur différente dans le calcul de la diversité du paysage, les plans n'étant pas souvent les mêmes. Ce phénomène peut être utilisé pour analyser des panoramas en comparant le jeu des valeurs obtenues sur des photos balayant l'horizon. Il faut remarquer également que la diversité paysagère qui vient d'être présentée est bien corrélée avec la diversité cartographique qui peut être calculée d'une manière analogue en plaçant une grille sur une carte écologique.

\section{BIBLIO GRAPHIE}

1. FO REMAN R., GODRON M., 1985. Landscape ecology. New York, NY, USA, Wiley, $619 \mathrm{p}$.

2. GODRON M., 1971. Essai sur une approche probabiliste de l'écologie des végétaux. Thèse d'Etat, Université Montpellier II, Montpellier, France, $250 \mathrm{p}$

3. GODRON M., POISSONET J., 1976. Essai d'expression de la biodiversité des paysages. In : IVe Symposium int. sur la biologie et l'écologie des paysages, Smolenice,Tchécoslovaquie. Bratislava, Tchécoslovaquie, Académie des Sciences, $12 \mathrm{p}$.

4. PAPILLON Y.,GODRON M., 1997. Distribution du lapin de garenne (O ryctolagus cuniculus) dans le Puy-de-D ome : I'apport de l'analyse des paysages. Game Wild., 14 : 303-324.

5. POISSONET J., FORGIARINI G., TOUTAIN B., TOURE I., 1997. Cartographie de la végétation pastorale pour le projet Almy Bahaïm d'hydraulique pastorale dans le Tchad oriental. Montpellier, France, Cirad-emvt, 135 p. (rapport N E98008)

Reçu le 13.7.99, accepté le 30.3.00

Figure 6 : diversités effectives et maximales des 16 paysages de l'est du Tchad. 


\section{Summary}

Godron M., Poissonet J., Daget Ph. Evaluation of the landscape diversity in African rangelands

The diversity of a landscape can be measured by an application of the theory of information to the analysis of a panorama cut out in plans according to geomorphological facets and vegetation types. Applications are proposed on tropical N orthern Africa landscapes.

Key words: Pastures - M easurement - M ethods - Landscape Tropical zone.

\section{Resumen}

Godron M., Poissonet J., Daget Ph. Evaluar la diversidad del paisaje de los pastizales africanos

La diversidad de un paisaje puede medirse mediante la aplicación de la teoría de la información a un análisis del panorama, seccionado en planes según las facetas geomorfológicas y los tipos de vegetación. Se proponen aplicaciones sobre los paisajes de Africa tropical septentrional.

Palabras clave: Pastizales - Medición - Métodos - Paisaje Zona tropical. 